\title{
CASE REPORT \\ Increase in interleukin-6 immediately after wheelchair basketball games in persons with spinal cord injury: preliminary report
}

\author{
T Kinoshita ${ }^{1}$, T Nakamura ${ }^{1}$, Y Umemoto ${ }^{1}$, D Kojima $^{1}$, T Moriki ${ }^{1}$, T Mitsui ${ }^{1}$, M Goto $^{1}$, Y Ishida $^{2}$ \\ and F Tajima ${ }^{1}$
}

\begin{abstract}
Study design: Case series.
Objectives: To investigate the effects of wheelchair basketball game on plasma interleukin-6 (IL-6), tumor necrosis factor- $\alpha$ (TNF- $\alpha$ ), C-reactive protein (CRP) and blood cell counts in persons with spinal cord injury (SCl).

Setting: The 2009 Mei-shin League of Wheelchair Basketball Games held at Wakayama, Japan.

Participants: Five wheelchair basketball players with SCI voluntarily participated in this study.

Interventions: Blood samples were taken approximately $1 \mathrm{~h}$ before the player warm-up for the game and immediately after the game. Main outcome measures: IL-6, TNF- $\alpha, \mathrm{CRP}$ and blood cell count were measured.

Results: Plasma IL-6 level and number of monocytes were significantly increased after the game, compared with pre-game measurements $(P<0.05)$. No changes were observed in other measurements. There was a significant relationship between increased IL-6 levels and accumulated play duration.

Conclusion: The lack of change in TNF- $\alpha$ and CRP levels suggested that the exercise-induced rise in IL- 6 was not related to exerciseinduced inflammatory response. Furthermore, the associated increase in the number of monocytes did not correlate with exerciseinduced IL-6 changes, negating monocytes as the source of IL-6.
\end{abstract}

Spinal Cord (2013) 51, 508-510; doi:10.1038/sc.2013.4; published online 12 February 2013

Keywords: myokines; physical fitness; wheelchair sports; cytokines

\section{INTRODUCTION}

The physical activity of daily living is inadequate for maintenance of proper physical fitness. ${ }^{1}$ Various sports activities are therefore recommended especially for wheelchair-bound handicapped individuals. Ludwig Guttmann started wheelchair basketball in 1944 through a rehabilitation program at Stoke Mandeville Hospital in England, by adapting the existing sport to wheelchair use. Today, wheelchair basketball is one of the major sports practiced by individuals with disability, with over 1000 players participating.

Recently, Pedersen et al. ${ }^{2}$ identified skeletal muscle as an endocrine organ that secretes cytokines and other peptides. Molecules mediating these endocrine effects are classified as 'myokines.' The first identified and most studied myokine is interleukin-6 (IL-6). IL-6 is markedly upregulated and released during the post-exercise period, and is known to enhance lipid oxidation, improve insulin-stimulated glucose uptake, and has anti-inflammatory effects. ${ }^{2,3}$ The magnitude of post-exercise rise in plasma IL-6 correlates with the exercise duration and intensity, the muscle mass involved in the mechanical work, and the endurance capacity of the muscle. ${ }^{2,3}$

Recently, we reported that arm crank ergometer exercise in laboratory settings induced an increase in plasma IL-6 in people with spinal cord injury (SCI). ${ }^{4}$ However, the study showed data generated from a laboratory-based setting, and thus the response of IL-6 to actual sport conducted in the field remains unknown at this stage. Several persons with SCI participated in the Mei-shin League of Wheelchair Basketball Games held at Wakayama City in 2009. We had the opportunity at that time to collect and analyze blood samples from these athletes. The present preliminary study investigated changes in plasma IL-6 immediately after wheelchair basketball game in persons with SCI.

\section{SUBJECTS AND METHODS}

\section{Subjects}

Five wheelchair basketball players with SCI voluntarily participated in this study. All subjects participated in the Mei-shin League of Wheelchair Basketball Games in 2009, and were healthy except for their SCI-related medical problems. Only five players consented to participate in this study as the other players considered such participation a distraction from the competition or could influence their performance. The study subjects were considered true representatives of the wheelchair basketball players participating in the games since they had partaken basketball training 2 days per 1 week for $>1$ year. The mean age of the subjects was $32.8 \pm 7.6$ years (mean \pm s.d.; range, $26-45$ years) The levels of spinal lesion and ASIA scale in the five subjects were T11, T11, T7, T12, T10, and C, C, A, C, C, respectively. The human ethics committee of Wakayama Medical University approved this study and written informed consent was obtained from each subject.

${ }^{1}$ Department of Rehabilitation Medicine, Wakayama Medical University, Wakayama city, Wakayama, Japan and ${ }^{2}$ Department of Forensic Medicine, Wakayama Medical University, Wakayama city, Wakayama, Japan

Correspondence: Dr F Tajima, Department of Rehabilitation Medicine, Wakayama Medical University, 811-1 Kimiidera, Wakayama city, Wakayama 641-8509, Japan. E-mail: fumi@wakayama-med.ac.jp

Received 6 July 2012; revised 8 January 2013; accepted 13 January 2013; published online 12 February 2013 


\section{Study protocol}

Blood sampling was conducted in every subject near the basketball court approximately $1 \mathrm{~h}$ before the game warm-up time. All subjects subsequently joined the game of basketball, the actual play duration time for each individual are listed in Table 1. The game of basketball includes four quarter per game, with $10 \mathrm{~min}$ for each quarter. Subject A played with the team during the first quarter, half of the third quarter and fourth quarter. Subjects B and E played only in the fourth quarter, while subjects $C$ and $D$ played in each of the four quarters. Immediately after the game, blood samples were again taken from all subjects to measure IL- 6 , tumor necrosis factor- $\alpha$ (TNF- $\alpha)$, C-reactive protein (CRP) and blood cell counts.

\section{Measurements}

IL- 6 and TNF- $\alpha$ were measured by enzyme-linked immunosorbent assay kit. CRP was measured by the latex nephelometry method. Total blood cell counts were determined using a cell counter. Each measurement was performed in duplicate.

\section{Statistical analysis}

Data were expressed as mean \pm s.d. Pre- and post-game results were compared using a Wilcoxon signed-rank test. Pearson's correlation was used to assess the statistical correlation between IL-6 and number of monocytes or IL- 6 and play duration. All statistical outcomes were considered significant at $P<0.05$. We calculated the statistical power and the necessary sample size to detect the difference as observed in this study. The statistical power was $59 \%$, and the sample size of 7 was necessary to achieve $80 \%$ power. For this reason, the results of this study should be viewed as preliminary in nature.

\section{RESULTS}

Plasma IL-6 and monocyte numbers were significantly increased immediately after exercise in all subjects (Table 1), but TNF- $\alpha$ and CRP showed no changes after the game. Red blood cell count, hematocrit, hemoglobin and white blood cell count were not different immediately after the game compared with pre-game results. The results showed a significant relationship between exercise-induced increases in IL-6 and accumulated play duration (Figure 1), although the IL- 6 changes did not correlate significantly with the increased number of monocytes.

\section{DISCUSSION}

The major findings of this study were as follows: (1) significant increases in plasma IL-6 levels and number of peripheral blood monocytes in individuals with SCI immediately after a single game of wheelchair basketball; (2) no significant change in TNF- $\alpha$ and CRP; and (3) the exercise-induced increase in IL-6 correlated significantly with accumulated play duration. We reported previously that IL-6 did

Table 1 Individual play time, plasma IL- 6 levels and number of monocytes before and immediately after wheelchair basketball game in persons with spinal cord injury

\begin{tabular}{|c|c|c|c|c|c|}
\hline \multirow[t]{2}{*}{ Subject } & \multirow[t]{2}{*}{ Play time (min) } & \multicolumn{2}{|c|}{$I L-6\left(p g \mathrm{~m}^{-1}\right)$} & \multicolumn{2}{|c|}{ Monocyte (per $\mu l)$} \\
\hline & & Pre-game & Post-game & Pre-game & Post-game \\
\hline$A$ & 25 & 0.49 & 1.74 & 112.0 & 218.4 \\
\hline$B$ & 10 & 0.74 & 1.26 & 194.7 & 226.8 \\
\hline C & 40 & 2.24 & 4.79 & 273.0 & 329.8 \\
\hline D & 40 & 0.77 & 2.62 & 620.4 & 804.0 \\
\hline$E$ & 10 & 1.31 & 2.12 & 690.0 & 725.2 \\
\hline Mean \pm s.d. & $25 \pm 14.14$ & $1.11 \pm 0.66$ & $2.5 \pm 1.29^{a}$ & $350 \pm 246$ & $461 \pm 266^{a}$ \\
\hline
\end{tabular}

Abbreviation: IL-6, interleukin-6.

a $P<0.05$, compared with pre-game level (by the Wilcoxon signed-rank test). not change during 5-h sitting at rest. ${ }^{4}$ Thus, this study presents the first evidence that playing a wheelchair basketball game induces a significant increase in IL-6, but not in TNF- $\alpha$ and CRP levels, in persons with SCI.

Recently, we reported that arm crank ergometer exercise in laboratory settings induced an increase in plasma IL-6 in people with SCI. ${ }^{4}$ In comparison, wheelchair basketball is intensive and contact sport. All players make maximal muscle contraction continuously to stop and move the wheelchair, in addition to playing the game. Thus, the exercise performed during wheelchair basketball game is quantitatively and qualitatively different from arm crank ergometer exercise in laboratory settings.

We demonstrated in a previous study that IL-6 did not increase significantly after 20-min arm crank ergometer exercise in subjects with cervical SCI. ${ }^{5}$ However, in this study, IL-6 increased significantly after about similar or shorter basketball play in SCI. These differences might be related to differences in the muscle mass of the participating subjects in the two studies, or to the period of training each group had undergone before the study.

The lack of change in TNF- $\alpha$ and CRP levels throughout the study suggested that the exercise-induced rise in IL- 6 was not related to exercise-induced inflammatory response. In addition, the associated increase in the number of monocytes did not correlate with exerciseinduced IL-6 changes, negating monocytes as the source of IL-6. Although the exact reason for the increased number of monocytes is not clear in this preliminary study, it could represent the effect of the intensive exercise on the hemodynamics and their release from the vascular endothelium. On the other hand, the significant relationship between IL-6 levels and accumulated play duration implicated contracting muscles as the source of increased IL-6 production. Pedersen et al. ${ }^{2,3}$ reported that IL-6 induced by muscle contraction promote the health of able-bodied persons. Extension of these results to our disabled individuals suggests that wheelchair basketball could promote the health of SCI subjects.

In this study, blood samples were obtained just before and immediately after the game. In other words, our results describe the acute changes in IL-6 after basketball game. However, we reported previously that an increase in IL- 6 peaked at $2 \mathrm{~h}$ after rather than immediately after arm crank ergometer exercise in people with SCI subjects. ${ }^{4}$ Thus, it is plausible that IL-6 continues to increase for a few

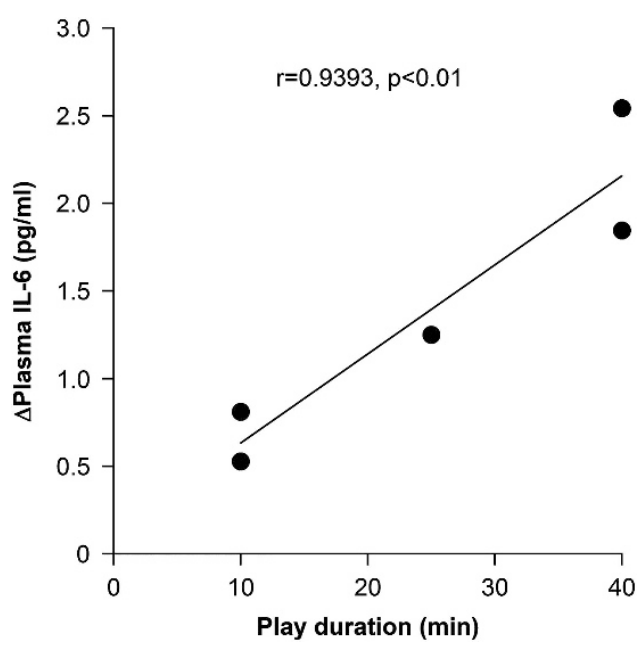

Figure 1 Relationship between exercise-induced IL-6 levels and accumulated play duration. The relationship was significant. 
hours after the end of wheelchair basketball game. Serial blood samples would allow examining the temporal profile of changes in IL-6 after playing the basketball game. However, this proved difficult in the actual setting, as the players were quarantined by their coach for the next game. Further studies are needed to confirm and support the study findings.

\section{CONCLUSION}

This study demonstrated a significant increase in plasma IL-6 levels immediately after the wheelchair basketball game in individuals with SCI. This finding suggests that wheelchair basketball could promote the health of SCI subjects through the activation of IL-6.

\section{CONFLICT OF INTEREST}

The authors declare no conflict of interest.

\section{ACKNOWLEDGEMENTS}

We thank Drs Midori Yamanaka, Yusuke Sasaki and Ken Kouda for the clinical assistance. We also thank Dr Faiq G Issa (www.word-medex.com.au) for the careful reading and editing of the manuscript.

1 Carlson KF, Wilt TJ, Taylor BC, Goldish GD, Niewoehner CB, Shamliyan TA et al. Effect of exercise on disorders of carbohydrate and lipid metabolism in adults with traumatic spinal cord injury: systematic review of the evidence. J Spinal Cord Med 2009; 32: 361-378.

2 Pedersen BK, Febbraio MA. Muscle as an endocrine organ: focus on muscle-derived interleukin-6. Physiol 2008; 88: 1379-1406.

3 Pedersen BK, Åkerström TCA, Nielsen AR, Fischer CP. Role of myokines in exercise and metabolism. J Appl Physiol 2007; 103: 1093-1098.

4 Umemoto Y, Furusawa K, Kouda K, Sasaki Y, Kanno N, Kojima D et al. Plasma IL-6 levels during arm exercise in persons with spinal cord injury. Spinal Cord 2011; 49: 1182-1187.

5 Kouda K, Furusawa K, Sugiyama H, Sumiya T, Ito T, Tajima F et al. Dose 20-min arm crank ergometer exercise increase plasma interleukin- 6 in individuals with cervical spinal cord injury? Eur J App/ Physiol 2012; 112: 597-604 